\title{
Response to letter re: Carotid atherosclerotic plaques standardized uptake values: methodological issues on reproducibility and accuracy
}

\author{
Nicola Giannotti ${ }^{1 *}$ (D, Martin J. O'Connell ${ }^{2,3}$, Shane J. Foley', Marie C. Galligan ${ }^{4}$, Peter J. Kelly ${ }^{3,5}$ \\ and Jonathan P. McNulty ${ }^{1}$
}

\section{Background}

Dear Editor,

We would like to thank Dr. Siamak Sabour for his letter and comments relating to our recently published article [1]. In the manuscript, an investigation was conducted on whether or not carotid atherosclerotic plaque standardized uptake values (SUVs) are consistent and reproducible across software packages; therefore, the purpose of the analysis performed was to measure the reproducibility, rather than validity, of SUV measurements between two software packages (OsiriX $\mathrm{MD}^{\circ}$ version 6.5.2, Pixmeo $\odot$ SARL, Geneva, Switzerland and AquariusNet iNtuitionTM version 4.4.11, TeraRecon, Foster City, CA, USA) (Table 1).

\section{Conclusions}

We acknowledge that the $p$ values reported in the manuscript previously submitted are dependent on the study sample size, and may not provide sufficient support of measurement reliability. Thus, we will now provide the intra-class coefficient (ICC) for the relevant variables (see Tables 2, 3, and 4) which was found to be supportive of our initial findings.

As expected, higher agreements (ICC) were found among SUV mean and maximum measurements. Effect size measurements also show that SUV max measurements were similar when compared (differences in mean values within the range: $0.02-0.05$ ).

\begin{tabular}{|c|c|c|c|}
\hline $\begin{array}{l}\text { Common carotid (CC) } \\
\text { and internal carotid (IC) } \\
\text { arteries }\end{array}$ & $\begin{array}{l}\text { TeraRecon } \\
\text { values } \\
\text { (mean } \pm \mathrm{SD} \text { ) }\end{array}$ & $\begin{array}{l}\text { Osirix values } \\
\text { (mean } \pm \text { SD) }\end{array}$ & $\begin{array}{l}\text { Difference } \\
\text { in values } \\
\text { (mean } \pm \text { SD) } \\
\end{array}$ \\
\hline SUV mean bifurcation left & $1.5 \pm 0.36$ & $1.58 \pm 0.43$ & $0.08 \pm 0.003$ \\
\hline SUV mean bifurcation right & $1.56 \pm 0.38$ & $1.67 \pm 0.46$ & $0.11 \pm 0.006$ \\
\hline SUV mean CC left & $1.08 \pm 0.59$ & $1.17 \pm 0.6$ & $0.09 \pm 0.004$ \\
\hline SUV mean CC right & $1.11 \pm 0.61$ & $1.23 \pm 0.64$ & $0.12 \pm 0.007$ \\
\hline SUV mean IC left & $1.56 \pm 0.45$ & $1.71 \pm 0.57$ & $0.15 \pm 0.01$ \\
\hline SUV mean IC right & $1.66 \pm 0.38$ & $1.72 \pm 0.44$ & $0.06 \pm 0.002$ \\
\hline SUV max bifurcation left & $2.54 \pm 0.65$ & $2.5 \pm 0.7$ & $0.04 \pm 0.008$ \\
\hline SUV max bifurcation right & $2.6 \pm 0.69$ & $2.62 \pm 0.73$ & $0.02 \pm 0.002$ \\
\hline SUV max CC left & $2.09 \pm 0.91$ & $2.06 \pm 0.88$ & $0.03 \pm 0.001$ \\
\hline SUV max CC right & $2.14 \pm 0.97$ & $2.16 \pm 0.99$ & $0.02 \pm 0.001$ \\
\hline SUV max IC left & $2.52 \pm 0.67$ & $2.57 \pm 0.84$ & $0.05 \pm 0.001$ \\
\hline SUV max IC right & $2.6 \pm 0.69$ & $2.58 \pm 0.74$ & $0.02 \pm 0.001$ \\
\hline SUV min bifurcation left & $0.71 \pm 0.3$ & $0.87 \pm 0.36$ & $0.16 \pm 0.012$ \\
\hline SUV min bifurcation right & $0.76 \pm 0.3$ & $0.94 \pm 0.38$ & $0.18 \pm 0.016$ \\
\hline SUV min CC left & $0.45 \pm 0.36$ & $0.56 \pm 0.46$ & $0.11 \pm 0.006$ \\
\hline SUV min CC right & $0.48 \pm 0.39$ & $0.62 \pm 0.48$ & $0.14 \pm 0.01$ \\
\hline SUV min IC left & $0.86 \pm 0.34$ & $1 \pm 0.43$ & $0.14 \pm 0.01$ \\
\hline SUV min IC right & $0.84 \pm 0.29$ & $1 \pm 0.34$ & $0.16 \pm 0.012$ \\
\hline
\end{tabular}

Table 1 SUV measurements (mean and standard deviation) by location and software together with differences (mean and

\footnotetext{
* Correspondence: Nicola.giannotti@ucd.ie

${ }^{1}$ Radiography and Diagnostic Imaging, School of Medicine, University

College Dublin, Dublin, Ireland

Full list of author information is available at the end of the article
} 
Table 2 ICC for SUVs mean with 95\% confidence intervals

\begin{tabular}{llll}
\hline Location & ICC & $\begin{array}{l}\text { Confidence limit } \\
\text { minimum }\end{array}$ & $\begin{array}{l}\text { Confidence limit } \\
\text { maximum }\end{array}$ \\
\hline SUV mean bifurcation left & 0.843 & 0.806 & 0.872 \\
SUV mean bifurcation right & 0.787 & 0.733 & 0.828 \\
SUV mean CC left & 0.906 & 0.887 & 0.922 \\
SUV mean CC right & 0.924 & 0.858 & 0.924 \\
SUV mean IC left & 0.79 & 0.713 & 0.842 \\
SUV mean IC right & 0.828 & 0.727 & 0.883
\end{tabular}

Table 3 ICC for SUVs max with 95\% confidence intervals

\begin{tabular}{llll}
\hline Location & ICC & $\begin{array}{l}\text { Confidence limit } \\
\text { minimum }\end{array}$ & $\begin{array}{l}\text { Confidence limit } \\
\text { maximum }\end{array}$ \\
\hline SUV max bifurcation left & 0.826 & 0.803 & 0.846 \\
SUV max bifurcation right & 0.817 & 0.793 & 0.838 \\
SUV max CC left & 0.83 & 0.808 & 0.85 \\
SUV max CC right & 0.891 & 0.803 & 0.891 \\
SUV max IC left & 0.752 & 0.699 & 0.795 \\
SUV max IC right & 0.791 & 0.721 & 0.838
\end{tabular}

Table 4 ICC for SUVs min with 95\% confidence intervals

\begin{tabular}{llll}
\hline Location & ICC & $\begin{array}{l}\text { Confidence limit } \\
\text { minimum }\end{array}$ & $\begin{array}{l}\text { Confidence limit } \\
\text { maximum }\end{array}$ \\
\hline SUV min bifurcation left & 0.627 & 0.494 & 0.773 \\
SUV min bifurcation right & 0.635 & 0.434 & 0.748 \\
SUV min CC left & 0.74 & 0.694 & 0.783 \\
SUV min CC right & 0.65 & 0.545 & 0.705 \\
SUV min IC left & 0.788 & 0.656 & 0.858 \\
SUV min IC right & 0.428 & 0.27 & 0.546 \\
\hline
\end{tabular}

Once again we thank Dr. Sabour for his contribution to the important discussion around SUV measurements across software packages.

\section{Authors' contributions}

NG, JMN, SF and PK designed the study. MOC inputted into PET-CT acquisition and analysis. NG collected, anonymized and analysed all data sets. NG, SF and JMN worked together on statistical analysis and data interpretation. NG led the write up of the manuscript. JMN and PK oversaw the study. However, all authors contributed to revising the manuscript and all approved the final manuscript.

\section{Competing interests}

The authors declare that they have no competing interests.

\section{Publisher's Note}

Springer Nature remains neutral with regard to jurisdictional claims in published maps and institutional affiliations.

\section{Author details}

${ }^{1}$ Radiography and Diagnostic Imaging, School of Medicine, University College Dublin, Dublin, Ireland. ²Department of Radiology, Mater Misericordiae University Hospital, Dublin, Ireland. ${ }^{3}$ School of Medicine, University College Dublin, Dublin, Ireland. ${ }^{4}$ UCD Clinical Research Centre,
School of Medicine, University College Dublin, Dublin, Ireland. ${ }^{5}$ Neurovascular Clinical Science Unit, Stroke Service and Department of Neurology, Mater University Hospital, Dublin, Ireland.

Received: 19 July 2017 Accepted: 19 July 2017

Published online: 07 September 2017

\section{Reference}

1. Giannotti N, O'Connell MJ, Foley SJ, Kelly PJ, McNulty JP. Carotid atherosclerotic plaques standardised uptake values: software challenges and reproducibility. EJNMMI Res. 2017;7(1):39. doi:10.1186/s13550-017-0285-0. Epub 2017 Apr 28.

\section{Submit your manuscript to a SpringerOpen ${ }^{\bullet}$ journal and benefit from:}

- Convenient online submission

- Rigorous peer review

- Open access: articles freely available online

- High visibility within the field

- Retaining the copyright to your article

Submit your next manuscript at $>$ springeropen.com 